\section{Prevalência e fatores associados à constipação intestinal: um estudo de base populacional em Pelotas, Rio Grande do Sul, Brasil, 2007}

\author{
Prevalence of intestinal constipation and associated \\ factors: a population-based study in Pelotas, \\ Rio Grande do Sul State, Brazil, 2007
}

\author{
1 Universidade Federal de \\ Pelotas, Pelotas, Brasil. \\ 2 Universidade Católica de \\ Pelotas, Pelotas, Brasil. \\ Correspondência \\ V. L. Collete \\ Universidade Federal de \\ Pelotas. \\ Rua José Trilho Otero Junior \\ 80 , Pelotas, $R S$ \\ 96020-120, Brasil. \\ vacollete@yahoo.com.br
}

\begin{abstract}
The aim of this study was to estimate the prevalence of intestinal constipation and associated factors in adults 20 years and older. A cross-sectional study was conducted in the rural area of Pelotas, Rio Grande do Sul State, Brazil, in 2007. Intestinal constipation was defined according to the Rome III criteria. The study began with a descriptive analysis, followed by bivariate and multivariate analyses using Poisson regression. Prevalence of intestinal constipation was 26.9\% (95\%CI: 25.1 28.8). Women had 2.5 times more constipation than men (36.8\% vs. 13.9\%). The adjusted analysis showed that risk factors for men were age (> 60 years), skin color (black/brown), and low economic status. Among women, age was inversely related, i.e., having a protective effect among the elderly. Intestinal constipation is common in this population. Associated factors were the same for men and women, except socioeconomic status, which showed no association in women.
\end{abstract}

Constipation; Gastrointestinal Motility; Adult Health
Vanessa Louise Collete 1,2

Cora Luiza Araújo 1

Samanta Winck Madruga ${ }^{1}$

\section{Introdução}

Constipação intestinal é a queixa digestiva mais comum na população geral, sendo responsável por cerca de 2,5 milhões de visitas médicas ${ }^{1}$, pelo gasto de vários milhões de dólares com laxantes e, indiretamente, por 92 mil hospitalizações anualmente nos Estados Unidos 2. Além disso, a constipação propriamente dita pode ser um sintoma inicial de doenças graves, como por exemplo, o câncer colorretal, que é o quinto câncer mais freqüente entre os homens e o quarto entre as mulheres no Brasil 3 .

As prevalências de constipação intestinal variam, principalmente, de acordo com o local e o critério diagnóstico utilizado. Dados da América do Norte mostraram uma variação entre $2 \%$ e $27 \%$ na prevalência dessa doença $4,5,6$, e nos países da Europa e Oceania entre 5\% e 35\% 7. No Brasil, não existem dados publicados de prevalência na população geral, visto que os estudos encontrados na literatura foram todos realizados em subgrupos, como lactentes 8 , adolescentes 9,10 e mulheres na menopausa 11 .

Desde 1980, a partir da publicação do primeiro consenso de Roma (http://www.romecriteria. org/, acessado em Set/2007) para doenças funcionais gastrintestinais, os estudos epidemiológicos sobre o tema têm usado tais critérios como definição de constipação intestinal. Antes disso, constipação era definida com base no número de evacuações semanais ou no auto-relato. 
A cronicidade dos sintomas, a falta de orientação terapêutica adequada e o uso abusivo de laxantes podem ter como conseqüências o surgimento de outros problemas como: doença diverticular do cólon, hemorróidas, fissuras anais e fecalomas com impactação fecal 12 .

Considerando-se a importância dessa queixa e a ausência de estudos populacionais em nosso país, o objetivo do presente estudo foi estimar a prevalência de constipação intestinal e avaliar a sua associação com possíveis fatores de risco.

\section{Métodos}

Com a finalidade de atender ao objetivo do estudo foi utilizado um delineamento transversal, de base populacional, incluindo os moradores do perímetro urbano de Pelotas, município da Região Sul do Brasil, com cerca de 340 mil habitantes. O período de realização do estudo foi entre novembro de 2007 e janeiro de 2008.

O processo de amostragem foi realizado em dois estágios. Inicialmente, os 404 setores censitários do município (Instituto Brasileiro de Geografia e Estatística, http://www.ibge.gov.br, acessado em Jun/2008) foram ordenados por renda média dos responsáveis pelo domicílio, sendo sorteados 126 setores, proporcionalmente ao tamanho, por meio de uma amostragem sistemática com intervalo. Em seguida, os domicílios pertencentes aos setores escolhidos foram classificados quanto ao status de ocupação em residencial, comercial ou desabitado, e eleitos apenas os residenciais. Após esse processo, uma nova amostragem sistemática com intervalo foi utilizada para sortear os domicílios de cada setor. Em cada domicílio, foram entrevistados todos os indivíduos com idade igual ou superior a 20 anos, exceto os portadores de doença física ou mental que os incapacitassem de responder ao questionário, ou aqueles institucionalizados.

Com o intuito de reduzir as taxas de não-respondentes, as entrevistadoras foram orientadas a realizar pelo menos três visitas a cada domicilio sorteado, em dias e horários diferentes. Para aqueles indivíduos que não foram encontrados ou mantiveram a negação em participar do estudo, o supervisor do trabalho de campo fez uma última tentativa de contato.

O tamanho da amostra foi calculado a fim de avaliar uma prevalência de constipação intestinal estimada de $15 \%$, considerando-se erro aceitável de dois pontos percentuais e nível de 95\% de confiança (IC95\%). Além disso, foram acrescidos $10 \%$ para não-respondentes e multiplicado o resultado por 1,5 a fim de ajustar para o efeito de delineamento amostral. Para o estudo das asso- ciações, a amostra foi calculada com a intenção de detectar razões de prevalência de pelo menos 1,5 significativo no nível de $5 \%$, considerandose pelo menos $10 \%$ de indivíduos expostos aos fatores em estudo, com $80 \%$ de poder estatístico, acrescidos $15 \%$ para controle de possíveis fatores de confusão. A amostra final necessária foi de 2.846 indivíduos.

Constipação intestinal foi definida de acordo com o consenso de Roma III ${ }^{13}$, que se baseia nos seis critérios: esforço ao evacuar, fezes endurecidas ou fragmentadas, sensação de evacuação incompleta, sensação de obstrução ou bloqueio anorretal, manobras manuais para facilitar as evacuações e menos de três evacuações por semana (Tabela 1). Os indivíduos foram orientados para que respondessem às perguntas sobre o funcionamento intestinal natural, ou seja, sem o uso de laxantes, chás ou qualquer outro tipo de ajuda.

A presença de dois ou mais desses critérios nos últimos seis meses caracterizou a presença de constipação intestinal, sendo que cada critério foi considerado positivo quando atingiu os pontos de corte mostrados a seguir: (1) esforço evacuatório em pelo menos $25 \%$ das defecações - resposta equivalente a "freqüentemente" (pergunta $\mathrm{A} \geq 2$ ); (2) fezes endurecidas ou fragmentadas em pelo menos $25 \%$ das defecações - resposta equivalente a "freqüentemente" (pergunta $B \geq 2$ ); (3) sensação de evacuação incompleta em pelo menos $25 \%$ das defecações - resposta equivalente a "algumas vezes" (pergunta C $\geq 1$ ); (4) sensação de obstrução/bloqueio anorretal em pelo menos $25 \%$ das defecações - resposta equivalente a "algumas vezes" (pergunta $\mathrm{D} \geq 1$ ); (5) manobras manuais para facilitar em pelo menos $25 \%$ das defecações - resposta equivalente a "algumas vezes" (pergunta $\mathrm{E} \geq 1$ ); e (6) menos que três evacuações por semana.

Avaliou-se ainda constipação auto-referida por meio da pergunta: "O(a) Sr.(a) tem intestino preso ou prisão de ventre?”. Essa pergunta foi aplicada para medir a concordância entre a definição segundo os critérios de Roma III 13 e a informação auto-referida pelo entrevistado. O nível de concordância foi medido usando-se o coeficiente kappa.

As variáveis independentes avaliadas foram: demográficas - sexo, idade e cor da pele (branca, parda, preta e outras, observadas pela entrevistadora); socioeconômicas - escolaridade (anos completos de estudo) e nível socioeconômico segundo a classificação da Associação Brasileira de Empresas de Pesquisa 14, em cinco categorias, sendo a categoria A considerada como de melhor nível; antropométricas - índice de massa corporal (IMC) calculado com base no peso e altura 
Instrumento para definição de constipação intestinal baseado no consenso de Roma III.

\begin{tabular}{|c|c|c|}
\hline Critério & Pergunta & Opções de respostas \\
\hline Esforço evacuatório & $\begin{array}{c}\text { (A) Desde seis meses atrás, com que } \\
\text { freqüência o(a) Sr.(a) teve de fazer força ou } \\
\text { esforço para fazer cocô? }\end{array}$ & $\begin{array}{l}\text { (0) Nunca ou raramente } \\
\text { (1) Algumas vezes } \\
\text { (2) Freqüentemente } \\
\text { (3) Maior parte das vezes } \\
\text { (4) Sempre }\end{array}$ \\
\hline Fezes endurecidas ou fragmentadas & $\begin{array}{l}\text { (B) Desde seis meses atrás, com que } \\
\text { freqüência o(a) Sr.(a) teve fezes duras, } \\
\text { endurecidas ou como se fossem bolinhas? }\end{array}$ & $\begin{array}{l}\text { (0) Nunca ou raramente } \\
\text { (1) Algumas vezes } \\
\text { (2) Freqüentemente } \\
\text { (3) Maior parte das vezes } \\
\text { (4) Sempre }\end{array}$ \\
\hline Sensação de evacuação incompleta & $\begin{array}{l}\text { (C) Desde seis meses atrás, com que } \\
\text { freqüência o(a) Sr.(a) teve a sensação de } \\
\text { evacuação incompleta, ou seja, mesmo após } \\
\text { ter feito cocô, permaneceu com vontade? }\end{array}$ & $\begin{array}{l}\text { (0) Nunca ou raramente } \\
\text { (1) Algumas vezes } \\
\text { (2) Freqüentemente } \\
\text { (3) Maior parte das vezes } \\
\text { (4) Sempre }\end{array}$ \\
\hline $\begin{array}{l}\text { Sensação de obstrução ou bloqueio } \\
\text { anorretal }\end{array}$ & $\begin{array}{l}\text { (D) Desde seis meses atrás, com que } \\
\text { freqüência o(a) Sr.(a) sentiu que as fezes não } \\
\text { conseguem passar, que estão trancadas ou } \\
\text { presas no ânus? }\end{array}$ & $\begin{array}{l}\text { (0) Nunca ou raramente } \\
\text { (1) Algumas vezes } \\
\text { (2) Freqüentemente } \\
\text { (3) Maior parte das vezes } \\
\text { (4) Sempre }\end{array}$ \\
\hline $\begin{array}{l}\text { Manobras manuais para facilitar a } \\
\text { evacuação }\end{array}$ & $\begin{array}{c}\text { (E) Desde seis meses atrás, com que } \\
\text { freqüência o(a) Sr.(a) teve de usar o dedo } \\
\text { para ajudar na saída das fezes? }\end{array}$ & $\begin{array}{l}\text { (0) Nunca ou raramente } \\
\text { (1) Algumas vezes } \\
\text { (2) Freqüentemente } \\
\text { (3) Maior parte das vezes } \\
\text { (4) Sempre }\end{array}$ \\
\hline Número de evacuações semanais & $\begin{array}{c}\text { (F) Quantas vezes por semana o(a) Sr.(a) } \\
\text { costuma fazer cocô? }\end{array}$ & __ vezes/semana \\
\hline
\end{tabular}

auto-referidos, e classificado segundo critério da Organização Mundial da Saúde 15; comportamentais - tabagismo, sendo considerado fumante aquele indivíduo que fuma um ou mais cigarros por dia, há mais de um mês, e ex-fumante aquele que não fuma há mais de um mês; atividade física - avaliada pela versão curta do Questionário Internacional de Atividade Física (IPAQ), sendo considerados sedentários os indivíduos cuja prática de atividade física no lazer foi inferior a 150 minutos por semana 16 .

Para a coleta de dados utilizou-se questionário padronizado e codificado. Estudos-piloto foram realizados para testar a compreensão do questionário. No controle de qualidade foi aplicada uma versão resumida do questionário em $10 \%$ da amostra. A concordância entre as respostas foi avaliada pelo coeficiente kappa.

O banco de dados foi elaborado no programa Epi Info 6.04b (Centers for Disease Control and
Prevention, Atlanta, Estados Unidos) e os dados foram duplamente digitados e submetidos à checagem de amplitude e consistência. A análise estatística foi realizada no programa Stata versão 9.0 (Stata Corp., College Station, Estados Unidos). O desenho amostral foi levado em conta em todas as análises dos dados, de forma a corrigir as estimativas da variabilidade para correlação intraconglomerado. As análises bi e multivariáveis foram realizadas por meio de regressão de Poisson com ajuste robusto da variância e resultados expressos como razões de prevalências 17 . A análise multivariável baseou-se no modelo hierárquico, composto por três níveis assim distribuídos: primeiro (mais distal) - variáveis demográficas; segundo - variáveis socioeconômicas; terceiro (mais proximal) - variáveis comportamentais e antropométricas. A significância estatística de cada variável no modelo foi avaliada por meio do teste de Wald. Para cada nível realizou-se re- 
gressão por eliminação retrógrada, descartando variáveis com valor $\mathrm{p} \geq 0,20$.

As análises ajustadas foram realizadas modelando os termos de interação significativos entre a variável sexo e as variáveis escolaridade e nível econômico. Optou-se por utilizar a estratificação, pois acredita-se que o presente estudo possui número de participantes suficiente e adequado para a realização de análise estratificada, evitando assim, a complexidade dos modelos.

$\mathrm{O}$ estudo foi submetido e aprovado pelo Comitê de Ética e Pesquisa da Universidade Federal de Pelotas. Consentimento informado por escrito foi obtido dos participantes previamente à entrevista.

\section{Resultados}

Dentre os 1.522 domicílios elegíveis para o estudo, 1.450 foram visitados, encontrando-se 3.180 indivíduos com idade igual ou superior a vinte anos. Desses, foram entrevistados 2.986 e excluídos 40 indivíduos que mostraram incapacidade para responder ao questionário, totalizando assim 2.946 participantes. Um total de $6,1 \%$ da amostra (184 indivíduos) constituíram-se como não-respondentes, sendo a maioria do sexo masculino (57\%) e com idade inferior a 60 anos (76\%).

No controle de qualidade foram entrevistados 300 indivíduos, para os quais se repetiu a questão sobre constipação auto-referida. O resultado do teste kappa de concordância foi de 0,85 , que é considerado bom.

O coeficiente de correlação intraclasse foi 0,003 , produzindo neste estudo um efeito de delineamento amostral para constipação intestinal de 1,2 .

As análises de modificação de efeito realizadas entre as variáveis independentes mostraram a existência de interação entre sexo e idade ( $\mathrm{p} \leq$ $0,001)$ e sexo e escolaridade $(\mathrm{p}=0,007)$.

A média de idade dos indivíduos da amostra foi de 44,5 anos, variando entre 20 e 98 anos. Na Tabela 2 pode-se observar que $57 \%$ dos entrevistados eram do sexo feminino e $80,4 \%$ tinham a cor da pele branca. Quanto à condição socioeconômica, cerca de $63 \%$ pertenciam ao nível C ou inferior. Mais de $45 \%$ da amostra referiram ter estudado pelo menos 9 anos completos. No que se refere às variáveis comportamentais, cerca de $26 \%$ dos indivíduos avaliados eram fumantes, e mais da metade deles foi classificada como sedentários. Quanto ao estado nutricional, 52,1\% apresentaram IMC superior a $25 \mathrm{~kg} / \mathrm{m}^{2}$.

A prevalência de constipação intestinal foi de 26,9\% (IC95\%: 25,1-28,8), sendo mais freqüente nas mulheres (37\%), nos indivíduos de cor da pele preta ou parda $(33,4 \%)$ e entre aqueles de nível socioeconômico D/E (32\%). Observou-se uma tendência de aumento da prevalência conforme diminui o nível econômico (Tabela 1).

A Figura 1 descreve a freqüência dos critérios utilizados na definição de constipação intestinal entre os indivíduos classificados como constipados $(n=793)$. Em ambos os sexos, os dois critérios que mais se destacaram foram sensação de evacuação incompleta e de obstrução anorretal. O número de evacuações semanais inferiores a três foi duas vezes mais freqüente entre as mulheres do que entre os homens constipados $(42,5 \%$ vs. $21,6 \%)$. A utilização de manobras manuais para facilitar a evacuação foi o critério menos citado entre homens e mulheres constipados, respectivamente, 9,1\% e $12,6 \%$.

Em relação ao número de critérios positivos entre aqueles classificados como constipados (Figura 2), observa-se que entre os homens a maioria apresentava apenas dois dentre os seis critérios avaliados, enquanto que entre as mulheres cerca de $40 \%$ apresentaram quatro ou mais critérios.

A prevalência de constipação auto-referida foi de $25,6 \%$ (IC95\%: 24,0-27,2), sendo 10,8\% (IC95\% 9,2-12,5) entre os homens e 36,9\% (IC95\%: 34,4-39,2) entre as mulheres. A concordância entre constipação auto-referida e segundo o consenso Roma III 13 foi avaliada segundo o teste kappa, sendo o coeficiente igual a 0,59, 0,41 e 0,61 , respectivamente, para homens e mulheres.

Entre os homens (Tabela 3) houve uma tendência de crescimento da prevalência de constipação conforme o aumento da idade, sendo cerca de duas vezes maior nos idosos em relação àqueles com idades entre 20 e 29 anos. Em relação à cor da pele, observou-se uma associação estatisticamente significativa com maiores prevalências $(R P=1,70)$ entre os indivíduos com a cor da pele preta/parda em relação àqueles de cor branca. Essa associação permaneceu significativa mesmo após ajuste para idade. Além dessa, a escolaridade mostrou uma tendência inversa significativa na análise bruta e perdeu efeito após ajuste para as variáveis do mesmo nível e acima. O nível econômico foi outra variável que apresentou associação inversa significativa com constipação intestinal na análise bruta, permanecendo associado na ajustada. O IMC, o tabagismo e o nível de atividade física não se mostraram associados ao desfecho.

Assim como entre os homens, as variáveis idade e cor da pele estiveram associadas com constipação intestinal entre as mulheres. Aquelas com a cor da pele preta/parda tiveram prevalências 
Descrição da amostra e prevalência de constipação intestinal em adultos de 20 anos ou mais. Pelotas, Rio Grande do Sul, Brasil, $2007(n=2.946$ *).

\begin{tabular}{|c|c|c|c|c|}
\hline \multirow[t]{2}{*}{ Variável } & \multicolumn{2}{|c|}{ Distribuição amostral } & \multicolumn{2}{|c|}{ Constipação intestinal } \\
\hline & $\mathrm{n}$ & $\%$ & Prevalência & IC95\% ** \\
\hline \multicolumn{5}{|l|}{ Sexo } \\
\hline Masculino & 1.269 & 43,0 & 13,9 & $11,7-16,0$ \\
\hline Feminino & 1.677 & 57,0 & 36,8 & $34,2-39,3$ \\
\hline \multicolumn{5}{|l|}{ Idade (anos) } \\
\hline $20-29$ & 710 & 24,1 & 27,7 & $24,3-31,1$ \\
\hline $30-39$ & 550 & 18,7 & 29,0 & $24,7-33,4$ \\
\hline $40-49$ & 592 & 20,1 & 23,8 & $20,1-27,5$ \\
\hline $50-59$ & 517 & 17,5 & 25,3 & $21,7-29,0$ \\
\hline$\geq 60$ & 577 & 19,6 & 28,4 & $24,6-32,2$ \\
\hline \multicolumn{5}{|l|}{ Cor da pele } \\
\hline Branca & 2.369 & 80,4 & 25,3 & $23,6-27,0$ \\
\hline Preta/Parda & 577 & 19,6 & 33,4 & $28,8-38,1$ \\
\hline \multicolumn{5}{|c|}{ Escolaridade (anos) } \\
\hline $0-4$ & 679 & 23,0 & 29,2 & $25,6-32,7$ \\
\hline $5-8$ & 931 & 31,6 & 27,0 & $23,8-30,1$ \\
\hline $9-12$ & 834 & 28,3 & 25,1 & $22,4-27,7$ \\
\hline 13 ou mais & 502 & 17,1 & 26,9 & $23,2-30,5$ \\
\hline \multicolumn{5}{|c|}{ Nível econômico (ABEP) } \\
\hline $\mathrm{A} / \mathrm{B}$ & 1.091 & 37,4 & 25,5 & $23,0-27,9$ \\
\hline$C$ & 1.357 & 46,5 & 26,4 & $24,0-28,8$ \\
\hline $\mathrm{D} / \mathrm{E}$ & 469 & 16,1 & 32,0 & $27,0-36,9$ \\
\hline \multicolumn{5}{|l|}{ Atividade física } \\
\hline Sedentário & 1.510 & 51,5 & 27,6 & $25,0-30,2$ \\
\hline Ativo & 1.422 & 48,5 & 26,4 & $24,1-28,6$ \\
\hline \multicolumn{5}{|l|}{ Tabagismo } \\
\hline Não-fumante & 1.540 & 52,3 & 27,1 & $25,1-29,2$ \\
\hline Fumante & 763 & 25,9 & 27,1 & $23,3-30,9$ \\
\hline Ex-fumante & 643 & 21,8 & 26,1 & $22,5-29,7$ \\
\hline \multicolumn{5}{|c|}{ Índice de massa corporal (kg/m²) } \\
\hline$<25,0$ & 1.277 & 47,9 & 27,6 & $24,9-30,3$ \\
\hline $25,0-29,9$ & 948 & 35,5 & 27,3 & $24,3-30,2$ \\
\hline 30,0 ou mais & 444 & 16,6 & 20,5 & $16,3-24,6$ \\
\hline
\end{tabular}

ABEP: Associação Brasileira de Empresas de Pesquisa.

* O valor máximo de informações perdidas foi de 276 (9,3\%) na variável índice de massa corporal;

** Intervalo de 95\% de confiança (IC95\%) ajustado para efeito de delineamento amostral.

cerca de $24 \%$ maiores do que as de cor branca. Entretanto, a idade comportou-se diferentemente entre as mulheres, mostrando uma tendência de diminuição das prevalências com o aumento da idade. As demais variáveis mostraram-se associadas, embora com razões de prevalências muito próximas de um, e sem significância estatística (Tabela 4).

A ingestão de alimentos como frutas, vegetais, iogurtes e chás, o uso de laxantes e a prática de atividade física foram algumas medidas utilizadas pelos entrevistados para auxiliar o funcionamento intestinal. Do total da amostra, 36\% (IC95\%: 33,7-38,5) referiram usar alguma ajuda. $\mathrm{O}$ uso entre as mulheres foi duas vezes maior do que entre os homens $(47,0 \%$ vs. $21,6 \%$, respectivamente, $\mathrm{p}<0,001)$. Entre esses indivíduos que referiam usar alguma ajuda, 22,4\% (IC95\%: 19,625,2 ) citaram o uso de laxantes. 
Figura 1

Prevalência dos critérios entre adultos constipados. Pelotas, Rio Grande do Sul, Brasil, 2007 ( $n=793)$.

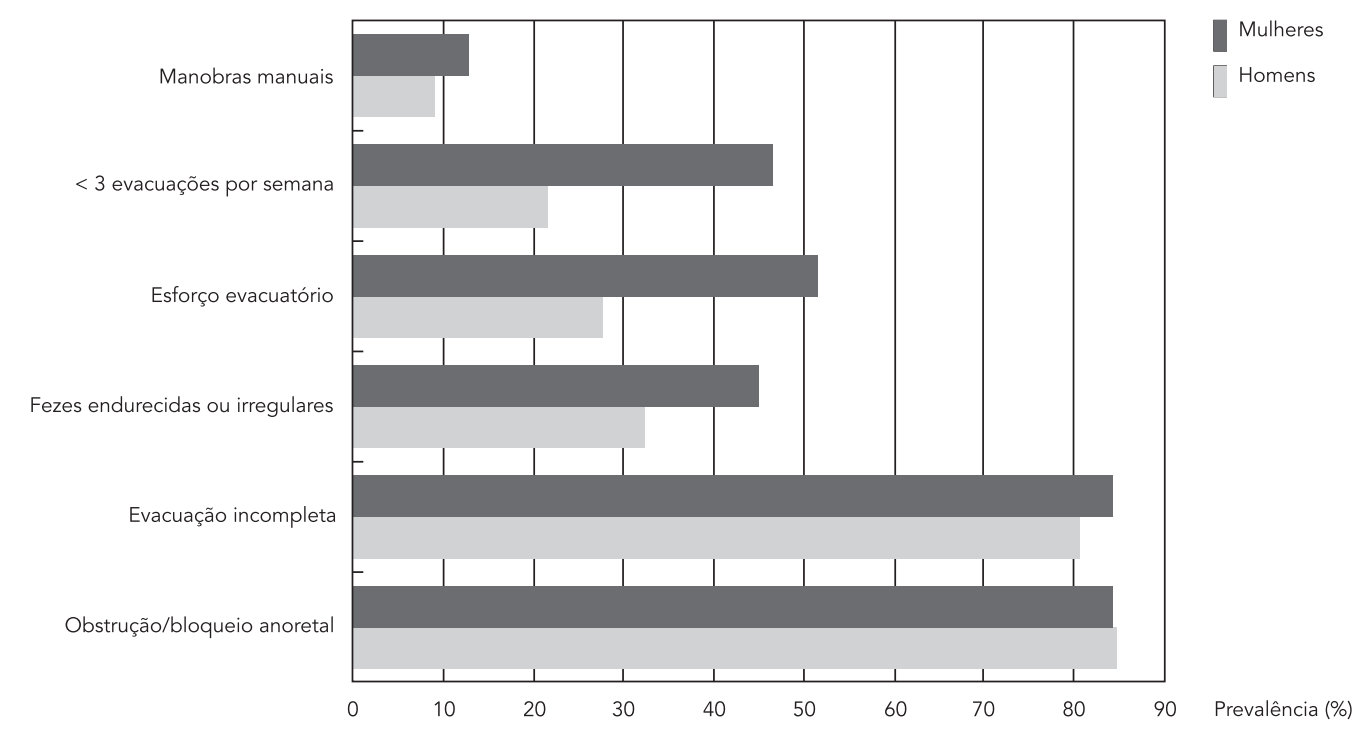

Figura 2

Proporção do número de critérios entre constipados conforme sexo. Pelotas, Rio Grande do Sul, Brasil, 2007 ( $n=793$ ).

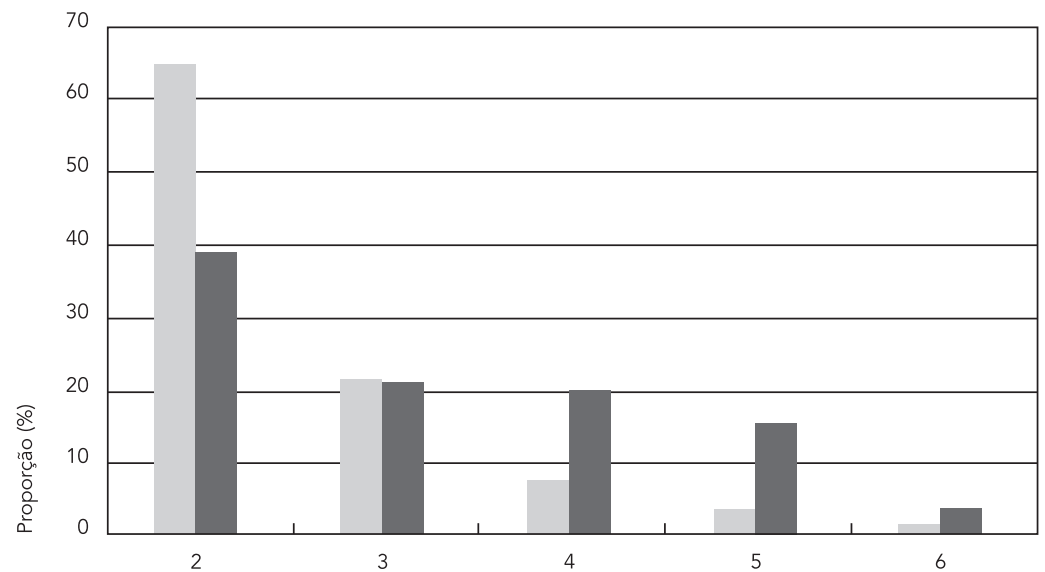

$\bigsqcup$ Homens

Mulheres 
Análises bruta e ajustada de constipação intestinal, e variáveis demográficas, socioeconômicas, antropométricas e comportamentais entre homens. Pelotas, Rio Grande do Sul, Brasil, 2007 ( $n=1.269)$.

\begin{tabular}{|c|c|c|c|c|c|c|}
\hline \multirow[t]{2}{*}{ Nível * } & \multirow[t]{2}{*}{ Variáveis } & \multirow[t]{2}{*}{ Prevalência } & \multicolumn{2}{|c|}{ Bruta } & \multicolumn{2}{|c|}{ Ajustada ** } \\
\hline & & & RP (IC95\%) & Valor de $\mathrm{p}$ & RP (IC95\%) & Valor de $p$ \\
\hline \multirow[t]{9}{*}{1} & Idade (anos) & & & 0,006 *** & & $0,004 * \star \star$ \\
\hline & 20-29 & 9,8 & 1,00 & & 1,00 & \\
\hline & $30-39$ & 15,2 & $1,54(1,01-2,33)$ & & $1,53(1,01-2,31)$ & \\
\hline & $40-49$ & 13,0 & $1,32(0,87-2,03)$ & & $1,31(0,87-1,99)$ & \\
\hline & $50-59$ & 12,6 & $1,28(0,80-2,06)$ & & $1,28(0,80-2,06)$ & \\
\hline & $\geq 60$ & 20,6 & $2,10(1,35-3,26)$ & & $2,18(1,40-3,39)$ & \\
\hline & Cor da pele & & & $0,01 \#$ & & $0,005 \#$ \\
\hline & Branca & 12,3 & 1,00 & & 1,00 & \\
\hline & Preta/Parda & 20,1 & $1,63(1,11-2,39)$ & & $1,70(1,18-2,46)$ & \\
\hline \multirow[t]{9}{*}{2} & Escolaridade (anos) & & & $0,003 * \star \star$ & & $0,33 * \star \star$ \\
\hline & 13 ou mais & 12,6 & 1,00 & & 1,00 & \\
\hline & $9-12$ & 8,1 & $0,65(0,40-1,02)$ & & $0,65(0,41-1,01)$ & \\
\hline & $5-8$ & 16,4 & $1,30(0,90-1,90)$ & & $1,15(0,76-1,74)$ & \\
\hline & $0-4$ & 18,6 & $1,48(0,96-2,30)$ & & $1,07(0,63-1,82)$ & \\
\hline & Nível econômico (ABEP) & & & 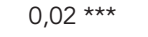 & & $0,04 * \star \star$ \\
\hline & $\mathrm{A} / \mathrm{B}$ & 12,1 & 1,00 & & 1,00 & \\
\hline & C & 13,0 & $1,08(0,77-1,50)$ & & $1,06(0,75-1,49)$ & \\
\hline & $\mathrm{D} / \mathrm{E}$ & 21,5 & $1,78(1,18-2,65)$ & & $1,63(1,10-2,41)$ & \\
\hline \multirow[t]{11}{*}{3} & Índice de massa corporal (kg/m²) & & & $0,69 * \star \star$ & & $0,83 * \star \star$ \\
\hline & $<25$ & 13,0 & 1,00 & & 1,00 & \\
\hline & $25-29,9$ & 15,3 & $1,18(0,88-1,58)$ & & $1,20(0,90-1,61)$ & \\
\hline & $\geq 30$ & 10,4 & $0,80(0,48-1,33)$ & & $0,84(0,51-1,38)$ & \\
\hline & Atividade física & & & $0,29 \#$ & & $0,51 \#$ \\
\hline & Ativa & 12,9 & 1,00 & & 1,00 & \\
\hline & Sedentária & 15,0 & $1,17(0,87-1,56)$ & & $1,10(0,82-1,47)$ & \\
\hline & Tabagismo & & & $0,12 \#$ & & 0,47 \# \\
\hline & Não-fumante & 11,5 & 1,00 & & 1,00 & \\
\hline & Ex-fumante & 15,8 & $1,37(0,98-1,92)$ & & $1,12(0,79-1,64)$ & \\
\hline & Fumante & 15,8 & $1,37(0,95-1,99)$ & & $1,26(0,79-1,64)$ & \\
\hline
\end{tabular}

ABEP: Associação Brasileira de Empresas de Pesquisa; RP: razão de prevalência; IC95\%: intervalo de $95 \%$ de confiança.

* Nível hierárquico da variável no modelo conceitual de análise;

** Níveis ajustados para todas as variáveis do mesmo nível e do nível superior;

*** Teste de Wald para tendência linear;

\# Teste de Wald para heterogeneidade.

\section{Discussão}

No Brasil, a constipação intestinal tem sido estudada em populações específicas 9,10,18. Este é o primeiro estudo de base populacional realizado no país com o objetivo de investigar a prevalência e fatores associados a essa queixa em adultos.

O critério utilizado para a definição de constipação intestinal foi escolhido por ser recomendado pelas diretrizes da Associação Americana de Gastroenterologia 19 e ainda muito empregado em estudos internacionais. O consenso de Roma para constipação funcional foi desenvolvido para padronizar a definição de constipação em estudos epidemiológicos, bem como para realizar o diagnóstico na prática clínica. Anteriormente à publicação de tais critérios, os estudos utilizavam como definição diagnóstica o número absoluto de evacuações semanais. Porém, sabese que isoladamente esse critério tem baixa sen- 
Tabela 4

Análises bruta e ajustada de constipação intestinal, e variáveis demográficas, socioeconômicas, antropométricas e comportamentais entre mulheres. Pelotas, Rio Grande do Sul, Brasil, 2007 ( $n=1.677$ ).

\begin{tabular}{|c|c|c|c|c|c|c|}
\hline \multirow[t]{2}{*}{ Nível * } & \multirow[t]{2}{*}{ Variáveis } & \multirow[t]{2}{*}{ Prevalência } & \multicolumn{2}{|c|}{ Bruta } & \multicolumn{2}{|c|}{ Ajustada ** } \\
\hline & & & RP (IC95\%) & Valor de $p$ & RP (IC95\%) & Valor de $p$ \\
\hline \multirow[t]{9}{*}{1} & Idade (anos) & & & 0,002 *** & & $0,003 * * *$ \\
\hline & 20-29 & 42,8 & 1,00 & & 1,00 & \\
\hline & $30-39$ & 40,2 & $0,94(0,77-1,13)$ & & $0,94(0,78-1,14)$ & \\
\hline & $40-49$ & 32,2 & $0,75(0,60-0,93)$ & & $0,76(0,61-0,93)$ & \\
\hline & $50-59$ & 34,9 & $0,81(0,67-0,99)$ & & $0,82(0,68-1,00)$ & \\
\hline & $\geq 60$ & 33,1 & $0,77(0,64-0,93)$ & & $0,78(0,65-0,93)$ & \\
\hline & Cor da pele & & & $0,002 \#$ & & $0,003 \#$ \\
\hline & Branca & 35,1 & 1,00 & & 1,00 & \\
\hline & Preta/Parda & 43,8 & $1,25(1,08-1,44)$ & & $1,24(1,07-1,42)$ & \\
\hline \multirow[t]{9}{*}{2} & Escolaridade (anos) & & & $0,49 * * *$ & & $0,84 * \star \star$ \\
\hline & 13 ou mais & 37,6 & 1,00 & & 1,00 & \\
\hline & $9-12$ & 37,8 & $1,00(0,85-1,19)$ & & $0,95(0,79-1,14)$ & \\
\hline & $5-8$ & 35,9 & $0,95(0,80-1,14)$ & & $0,96(0,78-1,19)$ & \\
\hline & $0-4$ & 36,1 & $0,96(0,80-1,14)$ & & $1,00(0,79-1,27)$ & \\
\hline & Nível econômico (ABEP) & & & $0,52 * \star \star$ & & $0,88 * \star \star$ \\
\hline & $A / B$ & 36,4 & 1,00 & & 1,00 & \\
\hline & C & 36,2 & $1,00(0,86-1,15)$ & & $0,96(0,83-1,11)$ & \\
\hline & $D / E$ & 39,2 & $1,08(0,90-1,30)$ & & $1,03(0,85-1,25)$ & \\
\hline \multirow[t]{11}{*}{3} & Índice de massa corporal (kg/m²) & & & $0,03 * \star \star$ & & $0,09 * \star \star$ \\
\hline & $<25$ & 38,3 & 1,00 & & 1,00 & \\
\hline & $25-29,9$ & 39,5 & $1,03(0,90-1,19)$ & & $1,07(0,92-1,24)$ & \\
\hline & $\geq 30$ & 28,8 & $0,75(0,60-0,94)$ & & $0,77(0,61-0,97)$ & \\
\hline & Atividade física & & & $0,58 \#$ & & $0,45 \#$ \\
\hline & Ativa & 37,6 & 1,00 & & 1,00 & \\
\hline & Sedentária & 36,3 & $0,97(0,85-1,09)$ & & $1,05(0,92-1,20)$ & \\
\hline & Tabagismo & & & $0,94 \#$ & & $0,94 \#$ \\
\hline & Não-fumante & 36,6 & 1,00 & & 1,00 & \\
\hline & Ex-fumante & 36,4 & $0,96(0,84-1,18)$ & & $0,97(0,80-1,17)$ & \\
\hline & Fumante & 37,5 & $1,02(0,87-1,20)$ & & $0,99(0,82-1,20)$ & \\
\hline
\end{tabular}

ABEP: Associação Brasileira de Empresas de Pesquisa; RP: razão de prevalência; IC95\%: intervalo de 95\% de confiança.

* Nível hierárquico da variável no modelo conceitual de análise;

** Níveis ajustados para todas as variáveis do mesmo nível e do nível superior;

*** Teste de Wald para tendência linear;

\# Teste de Wald para heterogeneidade.

sibilidade, visto que o indivíduo pode evacuar todos os dias à custa de grande esforço ou dificuldade 20. Outra definição ainda utilizada é o autorelato, o qual se considera um critério subjetivo que pode ser influenciado por costumes sociais e, segundo alguns autores, tem acurácia inferior quando comparada aos critérios baseados em sintomas 21.

A concordância entre os critérios de Roma III e auto-referida encontrada nesta investigação foi considerada moderada e semelhante àquelas en- contradas em outros estudos. Garrigues et al. 20 concluíram haver uma boa concordância entre constipação auto-referida e critérios de Roma I $(\mathrm{kappa}=0,68)$, e moderada entre auto-referida $\mathrm{e}$ Roma II (kappa = 0,55). Esses resultados sugerem que a informação auto-referida relatada pelo entrevistado de constipação, apesar de subjetiva e dependente de vários fatores, pode ser utilizada.

A prevalência de 26,9\% (IC95\%: 25,1-28,8) de constipação intestinal estimada pelo presente estudo está de acordo com aquelas encontradas 
em outras pesquisas de mesmo delineamento, realizadas em diferentes países 4,5,22,23. Mesmo assim, deve-se ter cautela ao comparar as prevalências encontradas baseando-se no uso dos critérios de Roma III com os anteriores, Roma I e II, tendo em vista as diferenças relativas ao número de sintomas e ao período recordatório da informação 13 .

A presença de sintomas específicos pode ser útil na diferenciação entre os subtipos de constipação. A presença de obstrução ou bloqueio anorretal sugere disfunção do andar pélvico como condição fisiopatológica associada ${ }^{19}$. Na presente investigação, em ambos os sexos, esse foi o sintoma mais freqüente entre aqueles indivíduos considerados com constipação. Segundo Garrigues et al. 20, esse sintoma e o esforço evacuatório foram os dois sintomas de melhor acurácia para confirmar a presença de constipação. De acordo com a pesquisa realizada por Lacerda Filho et al. 24, a sensação de evacuação incompleta, que foi o segundo critério mais freqüente no presente estudo em ambos os sexos, não tem o poder para definir um tipo específico de constipação intestinal.

Em relação aos fatores associados ao desfecho, o presente estudo mostrou uma prevalência de constipação duas vezes maior entre as mulheres. Outros trabalhos também relataram que a constipação é mais freqüente no sexo feminino, independentemente do critério diagnóstico adotado 25,26. Além disso, observou-se que as mulheres apresentam um número maior de critérios positivos dentre os seis, sendo, portanto, mais sintomáticas. Diferenças entre os sexos na motilidade intestinal e na percepção visceral já foram apontadas por alguns estudos como fatores associados a doenças gastrointestinais funcionais, incluindo a constipação intestinal 26 . Case \& Reid 27 mostraram que as alterações hormonais próprias do sexo feminino, tal como o aumento dos níveis de estrogênio durante a fase lútea do ciclo menstrual, estão associadas a um tempo de trânsito intestinal mais prolongado. Outro fator importante diz respeito às diferenças comportamentais entre os sexos. Desde a infância, o cuidado por parte das meninas em utilizar banheiros desconhecidos pode contribuir para que estas se tornem mais propensas a ignorar o reflexo evacuatório normal. Histórias de abuso sexual, físico e emocional, mais freqüentes no sexo feminino, também podem estar envolvidas em alguns distúrbios funcionais do sistema digestivo 28 .

A associação encontrada entre constipação intestinal e cor da pele, com maior prevalência nos indivíduos de cor preta ou parda, já tinha sido mostrada por outros autores 24,25. Higgins \& Johanson ${ }^{4}$ avaliaram múltiplos estudos sobre constipação realizados na América do Norte, e encontraram em média as prevalências $25 \%$ mais elevadas em indivíduos não-brancos, não sendo citada pelos autores a plausibilidade da associação encontrada.

Alguns autores sugerem que a redução na freqüência de movimentos intestinais estaria associada com anormalidades funcionais do cólon, as quais tendem a piorar com a idade 29 . No presente estudo, entre os homens verificou-se uma associação direta entre idade e constipação. Entretanto, nas mulheres o efeito foi oposto, com maiores prevalências nas faixas etárias de menor idade. Inquérito realizado por Dukas et al. 30 sobre constipação intestinal entre mulheres encontrou associação no mesmo sentido, ou seja, uma tendência estatisticamente significativa de diminuição da prevalência com o passar dos anos. Suspeita-se que essa tendência tenha seu início ainda na adolescência, uma vez que é nesse período que se iniciam todas as modificações hormonais e comportamentais que podem estar associadas à constipação. No Brasil, Oliveira et al. ${ }^{9}$ encontraram uma prevalência de $27,4 \%$ e $14,9 \%$ em adolescentes dos sexos feminino e masculino, respectivamente, sendo a diferença estatisticamente significativa $(\mathrm{p}=0,025)$.

A influência do status socioeconômico sobre a prevalência de constipação foi parcialmente consistente ao encontrado em diversos estudos 2,22,31. Embora utilizando diferentes métodos para classificar os indivíduos de acordo com a classe social, aqueles pertencentes aos níveis mais inferiores tiveram prevalências significativamente maiores de constipação intestinal. Bytzer et al. 32 , na Austrália, utilizando a classificação econômica própria daquele país, concluíram que tanto para homens quanto para mulheres existe uma clara tendência de aumento da prevalência de constipação intestinal conforme diminui o nível econômico. Esse achado pode ser devido à diferença nos hábitos alimentares conforme o status social. A literatura aponta para algumas evidências de que o consumo de fibras e alimentos saudáveis em geral é baixo na população como um todo, mas é ainda menor entre aqueles indivíduos pertencentes às classes menos favorecidas. Assim, o consumo pode mediar a associação do nível socioeconômico com maiores prevalências de constipação intestinal 33,34.

Em relação aos fatores comportamentais, dados populacionais apóiam a idéia de que indivíduos que praticam mais atividade física teriam menor freqüência de constipação 30,35 , principalmente devido ao fato de que a atividade física melhora a motilidade gastrintestinal, com mudanças proporcionais à quantidade de atividade exercida. No presente estudo não se verificou as- 
sociação entre atividade física e constipação em ambos os sexos. Tuteja et al. 36 , utilizando o método de aferição da atividade física (Kaiser Physical Activity Survey) e os critérios de Roma I para definir constipação, não encontraram diferença significativa na média total de atividade física em indivíduos com e sem constipação intestinal 36. Mesmo assim, as recomendações atuais para manejo de constipação intestinal propostas pela Associação Americana de Gastroenterologia sugerem a prática regular de atividade física 19. Quanto ao tabagismo, estudos têm mostrado que a nicotina, por meio de seu efeito adrenérgico, diminui o tempo de esvaziamento gástrico e aumenta a atividade motora intestinal. Tal efeito é diretamente proporcional ao número de cigarros fumados por dia, e pode ser confirmado por estudos que apontam a constipação como um dos sintomas mais referidos por indivíduos que abandonam o hábito de fumar ${ }^{30}$. Entretanto, no presente estudo, o tabagismo não se mostrou associado à constipação, possivelmente porque os fumantes foram classificados em apenas uma categoria.

Uma diminuição do tempo de trânsito intestinal e respostas secretoras alteradas poderiam explicar a associação direta entre os valores de IMC e constipação intestinal 30. Todavia, assim como na presente investigação, Talley et al. 37, utilizando o Bowel Disease Quetionnaire (BDQ) como definição de constipação intestinal, não encontraram associação estatisticamente significativa entre IMC e sintomas gastrintestinais.

Uma possível limitação do presente estudo é que não foi possível avaliar o hábito alimen- tar da população, e possivelmente o efeito desta variável tivesse influência sobre algumas das associações investigadas 38 . Por exemplo, dados mostram que indivíduos de baixo nível socioeconômico possuem hábito alimentar menos saudável 32. Mesmo reconhecendo a importância dessa variável, o delineamento transversal não seria o mais adequado para o estudo dessa associação, uma vez que é passível do viés de causalidade reversa.

Entretanto, algumas vantagens devem ser ressaltadas. Tem-se como principal ponto positivo o fato de este ser o primeiro estudo brasileiro de base populacional utilizando o consenso de Roma III. Associado a isso, o reduzido número de não-respondentes, diminuindo a possibilidade de ocorrência de viés de seleção.

Os resultados do presente estudo permitem, então, concluir que constipação intestinal é uma doença com elevada prevalência na população estudada, igualmente ao que ocorre em outros países. Foram confirmadas algumas associações com fatores de risco não modificáveis, como sexo, idade e cor da pele.

Baseando-se nos resultados obtidos no presente estudo, recomenda-se que outras pesquisas populacionais sejam realizadas com o intuito de avaliar outros possíveis fatores associados à constipação intestinal, como por exemplo, fatores comportamentais, principalmente o hábito alimentar e o uso de medicamentos, uma vez que o entendimento da distribuição da patologia entre os subgrupos é uma importante ferramenta na prática clínica na orientação do diagnóstico e conduta terapêutica para grupos específicos. 


\section{Resumo}

Estimar a prevalência de constipação intestinal e fatores associados entre adultos de 20 anos ou mais. Estudo transversal de base populacional conduzido na zona urbana de Pelotas, Rio Grande do Sul, Brasil, 2007. Constipação intestinal foi definida de acordo com os critérios de Roma III. Inicialmente foi realizada a análise descritiva e, posteriormente, utilizou-se Regressão de Poisson, as análises bi e multivariáveis. A prevalência de constipação intestinal encontrada foi de 26,9\% (IC95\%: 25,1-28,8). As mulheres apresentaram 2,5 vezes mais constipação que os homens (36,8\% vs. $13,9 \%$ ). Entre os homens, na análise ajustada, mostraram-se como fatores de risco idade maior que 60 anos, cor da pele preta/parda e menor nivel econômico. Entre as mulheres, idade teve relação inversa com o desfecho, apresentando efeito protetor entre as idosas. Constipação intestinal é relativamente comum na população estudada. Os fatores associados a essa doença foram os mesmos entre homens e mulheres, exceto o nível econômico, que não se mostrou associado entre as mulheres.

Constipação Intestinal; Motilidade Gastrointestinal; Saúde do Adulto

\section{Referências}

1. Sonnenberg A, Koch TR. Physician visits in the United States for constipation: 1958 to 1986. Dig Dis Sci 1989; 34:606-11.

2. Stewart WF, Liberman JN, Sandler RS, Woods MS, Stemhagen A, Chee E, et al. Epidemiology of constipation (EPOC) study in the United States: relation of clinical subtypes to sociodemographic features. Am J Gastroenterol 1999; 94:3530-40.

3. Instituto Nacional de Câncer. Incidência de câncer no Brasil, 2006. http://www.inca.gov.br/ estimativa/2006 (acessado em Ago/2007).

4. Higgins PD, Johanson JF. Epidemiology of constipation in North America: a systematic review. Am J Gastroenterol 2004; 99:750-9.

5. Adibi P, Behzad E, Pirzadeh S, Mohseni M. Bowel habit reference values and abnormalities in young Iranian healthy adults. Dig Dis Sci 2007; 52:1810-3.

6. Corazziari E. Definition and epidemiology of functional gastrointestinal disorders. Best Pract Res Clin Gastroenterol 2004; 18:613-31.

7. Peppas G, Alexiou VG, Mourtzoukou E, Falagas ME. Epidemiology of constipation in Europe and Oceania: a systematic review. BMC Gastroenterol 2008; 8:5.

\section{Colaboradores}

V. L. Collete foi responsável pela revisão da literatura, análise dos dados e redação do manuscrito. C. L. Araújo foi responsável pela coordenação da coleta de dados, orientação e revisão do manuscrito. S. W. Madruga colaborou na análise dos dados.
8. Aguirre AN, Vitolo MR, Puccini RF, Morais MB. Constipação em lactentes: influência do tipo de aleitamento e da ingestão de fibra alimentar. J Pediatr (Rio J.) 2002; 78:202-8.

9. Oliveira JN, Tahan S, Goshima S, Fagundes-Neto U, Morais MB. Prevalência de constipação em adolescentes matriculados em escolas de São José dos Campos, SP, e em seus pais. Arq Gastroenterol 2006; 43:50-4.

10. Carvalho EB, Vitolo MR, Gama CM, Lopez FA, Taddei JA, Morais MB. Fiber intake, constipation, and overweight among adolescents living in Sao Paulo City. Nutrition 2006; 22:744-9.

11. Oliveira SCM, Pinto-Neto AM, Góes JRN, Conde DM, Santos-Sá D, Costa-Paiva L. Prevalência e fatores associados à constipação intestinal em mulheres na pós-menopausa. Arq Gastroenterol 2005; 42:24-9.

12. Ambrogini Junior O, Miszputen SJ. Constipação intestinal crônica. In: Borges DR, Rothschild HA, organizadores. Atualização terapêutica. 20a Ed. São Paulo: Editora Artes Médicas; 2001. p. 411-3. 
13. Longstreth GF, Thompson WG, Chey WD, Houghton LA, Mearin F, Spiller RC. Functional bowel disorders. Gastroenterol 2006; 130:1480-91.

14. Associação Brasileira de Empresas de Pesquisa. Critério brasileiro de classificação econômica. São Paulo: Associação Brasileira de Empresas de Pesquisa; 2008.

15. Physical status: the use and interpretation of anthropometry. Report of a WHO Expert Committee. World Health Organ Tech Rep Ser 1995; 854:1-452.

16. American College of Sports Medicine Position Stand. The recommended quantity and quality of exercise for developing and maintaining cardiorespiratory and muscular fitness, and flexibility in healthy adults. Med Sci Sports Exerc 1998; 30: 975-91.

17. Barros AJ, Hirakata VN. Alternatives for logistic regression in cross-sectional studies: an empirical comparison of models that directly estimate the prevalence ratio. BMC Med Res Methodol 2003; 3:21.

18. Del Ciampo IR, Galvão LC, Del Ciampo LA, Fernandes MI. Prevalência de constipação intestinal crônica em crianças atendidas em unidade básica de saúde. J Pediatr (Rio J.) 2002; 78:497-502.

19. Locke 3rd GR, Pemberton JH, Phillips SF. AGA technical review on constipation. American Gastroenterological Association. Gastroenterol 2000; 119:1766-78.

20. Garrigues V, Galvez C, Ortiz V, Ponce M, Nos P, Ponce J. Prevalence of constipation: agreement among several criteria and evaluation of the diagnostic accuracy of qualifying symptoms and selfreported definition in a population-based survey in Spain. Am J Epidemiol 2004; 159:520-6.

21. Talley NJ. Definitions, epidemiology, and impact of chronic constipation. Rev Gastroenterol Disord 2004; 4 Suppl 2:S3-10.

22. Pare P, Ferrazzi S, Thompson WG, Irvine EJ, Rance L. An epidemiological survey of constipation in Canada: definitions, rates, demographics, and predictors of health care seeking. Am J Gastroenterol 2001; 96:3130-7.

23. Harris LA. Prevalence and ramifications of chronic constipation. Manag Care Interface 2005; 18:23-30.

24. Lacerda Filho A, Lima MJ, Magalhães MF, Paiva RA, Cunha-Melo JR. Chronic constipation - the role of clinical assessment and colorectal physiologic tests to obtain an etiologic diagnosis. Arq Gastroenterol 2008; 45:50-7.
25. Lembo A, Camilleri M. Chronic constipation. N Engl J Med 2003; 349:1360-8.

26. Chang L, Toner BB, Fukudo S, Guthrie E, Locke GR, Norton NJ, et al. Gender, age, society, culture, and the patient's perspective in the functional gastrointestinal disorders. Gastroenterol 2006; 130: 1435-46.

27. Case AM, Reid RL. Effects of the menstrual cycle on medical disorders. Arch Intern Med 1998; 158:1405-12.

28. Chen GD, Hu SW, Chen YC, Lin TL, Lin LY. Prevalence and correlations of anal incontinence and constipation in Taiwanese women. Neurourol Urodyn 2003; 22:664-9.

29. Harari D, Gurwitz JH, Minaker KL. Constipation in the elderly. J Am Geriatr Soc 1993; 41:1130-40.

30. Dukas L, Willett WC, Giovannucci EL. Association between physical activity, fiber intake, and other lifestyle variables and constipation in a study of women. Am J Gastroenterol 2003; 98:1790-6.

31. Sandler RS, Jordan MC, Shelton BJ. Demographic and dietary determinants of constipation in the US population. Am J Public Health 1990; 80:185-9.

32 Bytzer P, Howell S, Leemon M, Young LJ, Jones MP, Talley NJ. Low socioeconomic class is a risk factor for upper and lower gastrointestinal symptoms: a population based study in 15000 Australian adults. Gut 2001; 49:66-72.

33. Giskes K, Turrell G, Patterson C, Newman B. Socio-economic differences in fruit and vegetable consumption among Australian adolescents and adults. Public Health Nutr 2002; 5:663-9.

34. Shelton NJ. What not to eat: inequalities in healthy eating behaviour, evidence from the 1998 Scottish Health Survey. J Public Health (Oxf) 2005; 27: 36-44.

35. Muller-Lissner SA, Kamm MA, Scarpignato C, Wald A. Myths and misconceptions about chronic constipation. Am J Gastroenterol 2005; 100:232-42.

36. Tuteja AK, Talley NJ, Joos SK, Woehl JV, Hickam DH. Is constipation associated with decreased physical activity in normally active subjects? Am J Gastroenterol 2005; 100:124-9.

37. Talley NJ, Howell S, Poulton R. Obesity and chronic gastrointestinal tract symptoms in young adults: a birth cohort study. Am J Gastroenterol 2004; 99:1807-14.

38. Vitolo MR, Campagnolo PD, Gama CM. Fatores associados ao risco de consumo insuficiente de fibra alimentar entre adolescentes. J Pediatr (Rio J.) 2007; 83:47-52.

Recebido em 10/Abr/2009

Versão final reapresentada em 22/Mar/2010

Aprovado em 20/Mai/2010 